\title{
Response to Afatinib in a Patient with NSCLC Harboring Novel EGFR Exon 20 Insertion Mutations
}

This article was published in the following Dove Press journal: OncoTargets and Therapy

\author{
Ling $\operatorname{Lin}^{1}, *$ \\ Xiaomai Wu $\mathbb{1}^{1, *}$ \\ Shuangquan Yan' \\ Yefei Zhu' \\ Zhengqing Yan $^{2}$ \\ Dongqing $\mathrm{Lv}^{\prime}$ \\ Hongfei $\mathrm{Ge}^{3}$
}

'Department of Respiratory Medicine, Taizhou Hospital of Wenzhou Medical University, Taizhou, People's Republic of China; ${ }^{2}$ The Medical Department, 3D Medicines Inc., Shanghai, People's Republic of China; ${ }^{3}$ Department of Thoracic Surgery, Taizhou Hospital of Wenzhou Medical University, Taizhou, People's Republic of China

*These authors contributed equally to this work
Correspondence: Dongqing Lv; Hongfei Ge Email Lvdq@enzemed.com; gehf@enzemed.com
Purpose: Most epidermal growth factor receptor (EGFR) exon 20 insertion (ex20ins) mutations are resistant to tyrosine kinase inhibitors (TKIs). While some non-small cell lung cancer (NSCLC) patients harboring special subtypes of EGFR ex20ins still achieved clinical response after TKIs treatment, identifying special subtypes of EGFR ex20ins is helpful to find out NSCLC patients who can respond to TKIs.

Case Presentation: A 71-year-old non-smoker Chinese female was diagnosed with advanced lung adenocarcinoma harboring EGFR ex20ins (N771delinsKG). The patient received first-line afatinib (40 $\mathrm{mg}$ /day) therapy and a significant and substantial reduction in tumor size was observed subsequently. According to RESIST 1.1, a radiological partial response was achieved. The final progression-free survival was 10 months.

Conclusion: This is the first published case report of EGFR N771delinsKG lung adenocarcinoma, which highlighted the heterogeneity of clinical response to TKIs for EGFR ex20ins-mutant NSCLC. Such results need to be further investigated in prospective studies. Keywords: afatinib, lung adenocarcinoma, EGFR exon 20 insertions, next generation sequencing

\section{Introduction}

Epidermal growth factor receptor (EGFR) tyrosine kinase inhibitors (TKIs) have improved the pharmacological management of lung cancer significantly and become the standard first-line treatment for EGFR-mutant non-small cell lung cancer (NSCLC). ${ }^{1}$ The percentage of lung adenocarcinoma with EGFR mutations are about $10 \%$ and $50 \%$ in Western countries and East Asians, respectively. The classic EGFR mutations, including EGFR del in exon 19 and the L858R in exon 21, account for approximately $90 \%$ of all $E G F R$ mutation-positive cases. The lung adenocarcinoma harboring such classic EGFR mutations is usually sensitive to TKIs. The remaining $10 \%$ of EGFR mutations are uncommon EGFR alterations within exons 18-21. Among these uncommon mutations, about 50\% are EGFR exon 20 insertions (ex20ins). ${ }^{2}$ Until now, some preclinical and clinical studies have explored the clinical response to TKIs in NSCLC with EGFR ex20ins mutations. Since EGFR ex20ins mutations produced steric hindrance and activated EGFR without markedly decreasing affinity for ATP or enhancing affinity for TKIs, NSCLC harboring EGFR ex20ins mutations were usually resistant to the first and second generation TKIs. A combined post hoc analysis of LUX-Lung 2, LUX-Lung 3, and LUX-Lung 6 trails revealed that 23 NSCLC patients with EGFR ex20ins treated with afatinib had an objective response (ORR) of $8.7 \%$ and a median 


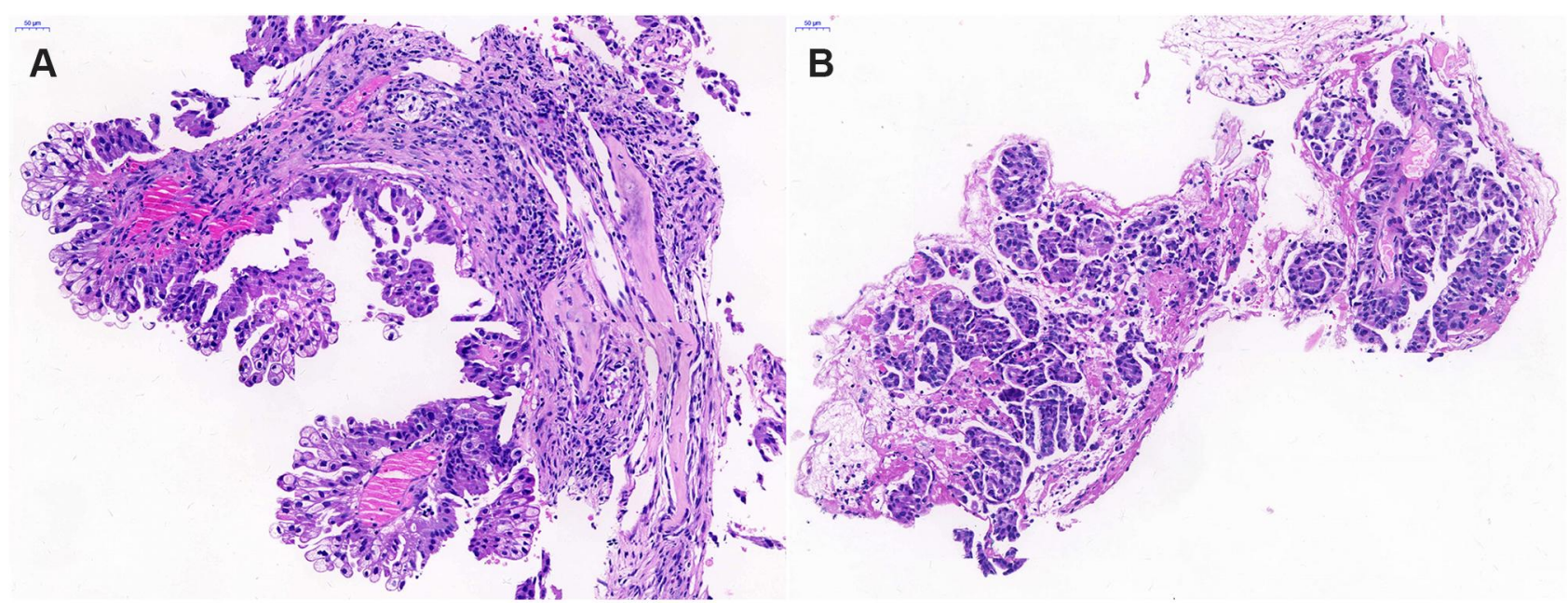

Figure I Hematoxylin-eosin (HE) staining for pathological diagnosis in (A) right and (B) left pulmonary lesions showing lung adenocarcinoma.

progression-free survival (PFS) of 2.7 months. ${ }^{3}$ In another Chinese real world study, 165 advanced EGFR ex20ins NSCLC patients were included to compare the clinical outcomes of chemotherapy and all generation TKIs. The median PFS was 6.4 for chemotherapy and 2.9 months for all generation TKIs. ${ }^{4}$ Considering the aforementioned unsatisfactory results, TKIs seems not to be a good choice for patients harboring $E G F R$ ex20ins. Until now, there are still no established molecular targeted drugs for NSCLC patients with $E G F R$ ex20ins.

However, to the best of our knowledge, a few reported cases also suggested that some subtypes of EGFR ex20ins could benefit from TKIs. For example, Yasuda et $\mathrm{al}^{5}$, and Voon et $\mathrm{al}^{6}$ reported the NSCLC patients harboring EGFR A763_764insFQEA demonstrated tumor regression and stable disease (SD) following erlotinib treatment, respectively. Chan et $\mathrm{al}^{7}$ reported a patient with EGFR A767_S768insSVA exhibited SD for 25 months with afatinib treatment. A patient with EGFR A767delinsASVD achieved partial response (PR) with afatinib treatment and PFS of 7.4 months. ${ }^{8}$ Jänne et $\mathrm{al}^{9}$ reported one patient harboring EGFR D770delinsGY, who achieved PR after dacomitinib treatment. Another patient harboring EGFR S768_D770dup could respond to osimertinib with PFS of over 5 months. ${ }^{10}$ In a retrospective study, six patients harboring EGFR ex20ins received osimertinib with median PFS for 6.2 months. Among them, four patients (EGFR A767_V769dup, S768_D770dup, D770_N771insG, and A763_Y764insFQEA\&T790M) achieved PR, and two cases (S768_D770dup and D770_N771insG) received SD. ${ }^{11}$ These cases suggested that some special subtypes of EGFR ex20ins mutations might respond to TKIs. Identifying and distinguishing subtypes of $E G F R$ ex20ins, which respond to TKIs, is an important issue for NSCLC patients. Herein, we presented a novel EGFR ex20ins (N771delinsKG) patient who could benefit from afatinib treatment.

\section{Case Presentation}

A 71-year-old non-smoker Chinese female was diagnosed with lung adenocarcinoma with multiple metastases in bilateral lung and mass in the right chest with osteolytic bone destruction in the sixth rib in May 2019 (Figure 1). The tumor node metastasis (TNM) classification of this patient was T4N3M1. Before treatment, her tumor DNA extracted from tissue was subjected to DNA sequencing analysis by nextgeneration sequencing (NGS). The sequencing results in Figure 2 suggested that the patient had a novel EGFR ex20ins (N771delinsKG) mutation. Based on the NGS results, the patient started to receive first-line treatment with afatinib (40 mg/day) in July 2019. Before the treatment, the computed tomography (CT) scans indicated that the size of tumor lesions located in the left lower lung and right chest were $62.6 \mathrm{~mm} \times 60.9 \mathrm{~mm}$ and $58.3 \mathrm{~mm} \times 46.7 \mathrm{~mm}$, respectively (Figure 3A). In addition, multiple small nodules appeared in bilateral lungs. After one month of afatinib treatment, the size of the lesion in the left lower lung reduced markedly and that of the tumor lesion in the right lung was stable (Figure 3B). After four months of treatment (November 2019), the size of these lesions further decreased. As shown in Figure 3C, one lesion in the left lower lung became obscure and unmeasurable, and the size of another lesion in the right chest was decreased to $43.7 \mathrm{~mm} \times 37.2 \mathrm{~mm}$. Notably, the size of multiple small nodules in bilateral lungs 


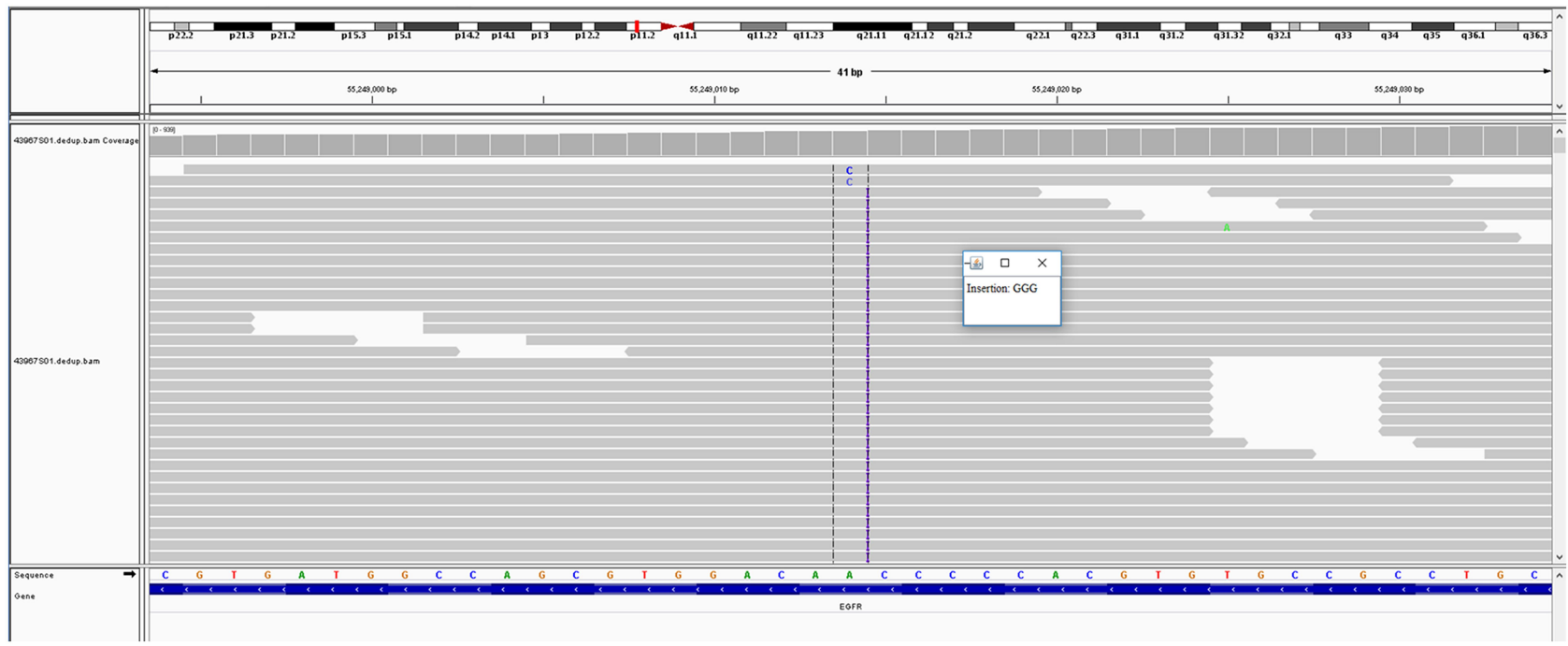

Figure 2 Molecular analysis of gene detection. The integrative genomics viewer snapshot of EGFR N77/delinsKG (c.23।2_23।3insGGG) by next-generation sequencing (NGS).

also significantly reduced after treatment. According to the response evaluation criteria in solid tumors (RESIST 1.1), she achieved a radiological PR. No grade three or higher adverse events (AEs) occurred. The treatment-related AEs associated with afatinib were grade 1 or 2 diarrhea, skin rash, paronychia, and stomatitis without treatment interruption. These AEs occurred during the first month of afatinib treatment and relieved with corresponding symptomatic treatments. Until May 2020, progressive disease was observed due to the enlarged tumor lesions. The final PFS was 10 months.

\section{Ethics Statement}

This case was approved by the Medical Ethics Committee of the Taizhou Hospital of Wenzhou Medical University. Written informed consent was obtained from the patient for publishing the case details and accompanying images.

\section{Discussion}

In this case, the Chinese female with advanced lung adenocarcinoma harboring a novel EGFR N771delinsKG achieved PR and the PFS exceeded 8 months. Such exciting results highlighted the heterogeneity of clinical response to TKIs for EGFR ex20ins. ${ }^{12,13}$ More than $90 \%$ of EGFR ex20ins are located behind the C-helix of the EGFR kinase domain (S768 to V774). Especially, V769_D770insASV and EGFR D770_N771insSVD are common EGFR ex20ins mutations. Less than $10 \%$ of all $E G F R$ ex20ins mutations occurred within the C-helix (E762 to M766). ${ }^{4,14,15}$
It was reported that EGFR A763_Y764insFQEA, which occurred within the C-helix of EGFR, was associated with sensitivity to available TKIs. ${ }^{5,6}$ Besides, most $E G F R$ ex20ins mutations were resistant to available TKIs. In 2013, Yasuda et $\mathrm{al}^{4}$ reported the first crystal structure of a representative EGFRD770_N771insNPG, which provided valuable insights into understanding the relationship between EGFR ex20ins and EGFR activation or TKIs resistance. It revealed EGFR D770_N771insNPG located towards the $\mathrm{C}$-terminal end of the C-helix, which was a key regulatory factor that activated EGFR via rotating it to form active conformation. Moreover, it pointed out that EGFR D770_N771insNPG was able to activate EGFR without decreasing ATP affinity or enhancing the affinity of the TKIs markedly. Another research involving 3D modeling of EGFR D770_N771insNPG suggested that such an alteration could result in a prominent shift of the C-helix and phosphate-binding loop (p-loop) and subsequently restrict the size of the drug-binding pocket. ${ }^{16}$ Considering that, EGFR ex20ins located in the p-loop of EGFR seems to be associated with insensitivity to TKIs. However, there are still some cases of EGFR ex20ins, which, located within p-loop, achieved clinical response to TKIs. For example, Jänne et $\mathrm{al}^{9}$ reported that one patient harboring EGFR D770delinsGY achieved PR after dacomitinib therapy. Three patients (EGFR A767_V769dup, S768_D770dup, and D770_N771insG) achieved PR and two cases (S768_D770dup and D770_N771insG) received SD during osimertinib treatment. ${ }^{11}$ In this case EGFR N771delinsKG also 

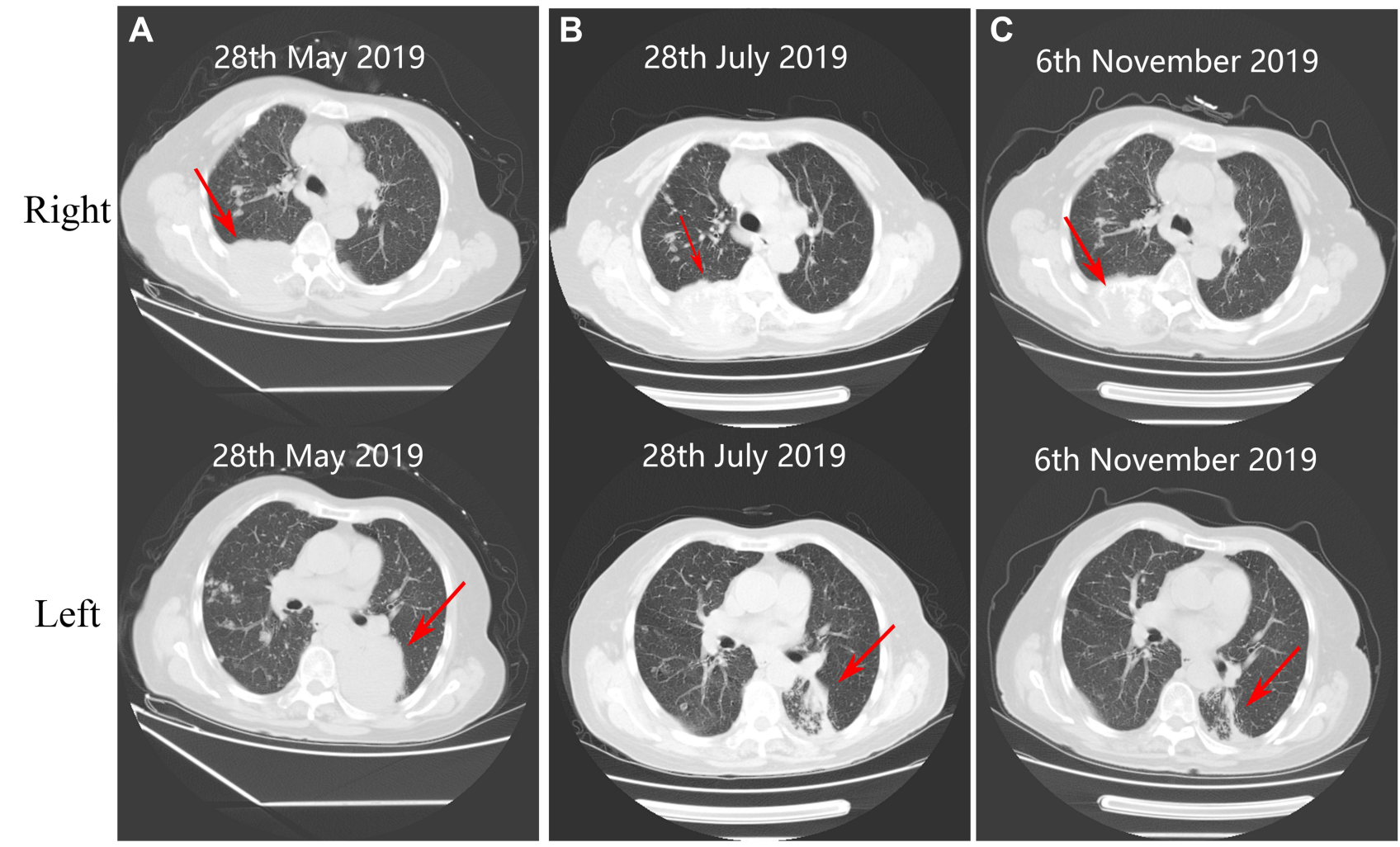

Figure 3 Computed tomography (CT) scans at different time. (A) Baseline imaging (before treatment) of right and left pulmonary lesions, respectively. After (B) I month (July 28, 2019) and (C) 4 months (November 6, 2019) of treatment, the mass in the left pulmonary lesion reduced significantly and the right pulmonary lesion decreased slightly, compared with that of baseline imaging.

occurred in the p-loop region and demonstrated exciting clinical response to second generation TKI afatinib. By the way, the rare EGFR ex20ins N771delinsKG in the present case was not described previously. Considering such exciting results, EGFR N771delinsKG might be attributed to a special subtype of EGFR ex20ins that was sensitive to afatinib. Additional in vitro and structural studies are needed to explain why some subtypes but not all respond to TKIs.

Given the significant molecular heterogeneity of $E G F R$ ex20ins, it would be important to identify the EGFR subtypes who respond to TKIs. The position, the size, and subtype of inserted amnio acids in EGFR ex20ins might confer the clinical heterogeneity to TKIs. With the development of NGS technology, more special mutation types of EGFR ex20ins would be identified due to its high accuracy, high throughput, and rich information content. In this case, the identification of EGFR ex20ins N771delinsKG benefited from the usage of NGS.

Considering the nature of the case report, some important issues still need to be studied. It is necessary to explore the potential mechanism for EGFR ex20ins- mutant NSCLC patients, who respond to TKIs. In addition, the long-term clinical durability of TKIs towards lung carcinoma harboring EGFR ex20ins remain unclear. To our best knowledge, several clinical trials have been carryied out to study TKIs on NSCLC with EGFR ex20ins, and some of them demonstrated preliminary promising activity. ${ }^{10,17,18}$ With the development of various TKIs, identifying and distinguishing novel subtypes of $E G F R$ ex20ins might be more crucial for EGFR mutant NSCLC to choose suitable agents.

\section{Conclusion}

Overall, we first reported a lung adenocarcinoma patient harboring a novel EGFR ex20ins mutation (N771delinsKG) benefited from afatinib treatment. Our findings highlighted the heterogeneity of clinical response to TKIs in EGFR ex20ins-mutant NSCLC. Further investigations need to be carried out to confirm these results.

\section{Funding}

The authors thank the patient for his participation and his agreement to the publication of the report. This work was 
supported by the Medical Science and Technology Project Foundation of Zhejiang Province of China (2015KYB439) and Scientific Research Fund of Taizhou Science and Technology Agency (1601KY05 and 1801KY19).

\section{Disclosure}

Zhengqing Yan is an employee of 3D Medicines Inc. The author reports no other potential conflicts of interest in this work.

\section{References}

1. Loong HH, Kwan SS, Mok TS, Lau YM. Therapeutic strategies in EGFR mutant non-small cell lung cancer. Curr Treat Options Oncol. 2018;19(11):58. doi:10.1007/s11864-018-0570-9

2. Sharma SV, Bell DW, Settleman J, Haber DA. Epidermal growth factor receptor mutations in lung cancer. Nat Rev Cancer. 2007;7 (3): $169-181$

3. Yang JCH, Sequist LV, Geater SL, et al. Clinical activity of afatinib in patients with advanced non-small-cell lung cancer harbouring uncommon EGFR mutations: a combined post-hoc analysis of LUX-lung 2, LUX-lung 3, and LUX-lung 6. Lancet Oncol. 2015;16(7):830-838. doi:10.1016/S1470-2045(15)00026-1

4. Yang G, Li J, Xu H, et al. EGFR exon 20 insertion mutations in Chinese advanced non-small cell lung cancer patients: molecular heterogeneity and treatment outcome from nationwide real-world study. Lung Cancer. 2020.

5. Yasuda H, Park E, Yun $\mathrm{CH}$, et al. Structural, biochemical, and clinical characterization of epidermal growth factor receptor (EGFR) exon 20 insertion mutations in lung cancer. Sci Transl Med. 2013;5 (216):216ra177. doi:10.1126/scitranslmed.3007205

6. Voon PJ, Tsui DW, Rosenfeld N, Chin TM. EGFR exon 20 insertion A763-Y764insFQEA and response to erlotinib-Letter. Mol Cancer Ther. 2013;12(11):2614-2615. doi:10.1158/1535-7163.MCT-13-0192

7. Chan RT. Afatinib for an EGFR exon 20 insertion mutation: a case report of progressive stage IV metastatic lung adenocarcinoma with 54 months' survival. Asia Pac J Clin Oncol. 2018;14(Suppl 1):7-9. doi:10.1111/ajco.12853
8. Cai Y, Wang X, Guo Y, et al. Successful treatment of a lung adenocarcinoma patient with a novel EGFR exon 20-ins mutation with afatinib: a case report. Medicine. 2019;98(1):e13890. doi:10.1097/ MD.0000000000013890

9. Janne PA, Boss DS, Camidge DR, et al. Phase I dose-escalation study of the pan-HER inhibitor, PF299804, in patients with advanced malignant solid tumors. Clin Cancer Res. 2011;17(5):1131-1139. doi:10.1158/1078-0432.CCR-10-1220

10. Piotrowska Z, Fintelmann FJ, Sequist LV, Jahagirdar B. Response to osimertinib in an EGFR exon 20 insertion-positive lung adenocarcinoma. $J$ Thorac Oncol. 2018;13(10):e204-e6. doi:10.1016/j.jtho.20 18.05.017

11. Fang W, Huang Y, Hong S, et al. EGFR exon 20 insertion mutations and response to osimertinib in non-small-cell lung cancer. $B M C$ Cancer. 2019;19(1):595. doi:10.1186/s12885-019-5820-0

12. Kobayashi Y, Mitsudomi T. Not all epidermal growth factor receptor mutations in lung cancer are created equal: perspectives for individualized treatment strategy. Cancer Sci. 2016;107(9):1179-1186.

13. Arcila ME, Nafa K, Chaft JE, et al. EGFR exon 20 insertion mutations in lung adenocarcinomas: prevalence, molecular heterogeneity, and clinicopathologic characteristics. Mol Cancer Ther. 2013;12 (2):220-229. doi:10.1158/1535-7163.MCT-12-0620

14. Vyse S, Huang PH. Targeting EGFR exon 20 insertion mutations in non-small cell lung cancer. Signal Transduct Target Ther. 2019;4 (1):5. doi:10.1038/s41392-019-0038-9

15. Yasuda H, Kobayashi S, Costa DB. EGFR exon 20 insertion mutations in non-small-cell lung cancer: preclinical data and clinical implications. Lancet Oncol. 2012;13(1):e23-e31. doi:10.1016/ S1470-2045(11)70129-2

16. Robichaux JP, Elamin YY, Tan Z, et al. Mechanisms and clinical activity of an EGFR and HER2 exon 20-selective kinase inhibitor in non-small cell lung cancer. Nat Med. 2018;24(5):638-646. doi:10.1038/s41591-018-0007-9

17. Fang W, Huang Y, Gan J, Shao YW, Zhang L. Durable response of low-dose afatinib plus cetuximab in an adenocarcinoma patient with a novel EGFR exon 20 insertion mutation. J Thorac Oncol. 2019;14 (10):e220-e1. doi:10.1016/j.jtho.2019.05.023

18. Hasegawa H, Yasuda H, Hamamoto J, et al. Efficacy of afatinib or osimertinib plus cetuximab combination therapy for non-small-cell lung cancer with EGFR exon 20 insertion mutations. Lung Cancer. 2019;127:146-152. doi:10.1016/j.lungcan.2018.11.039
OncoTargets and Therapy

\section{Publish your work in this journal}

OncoTargets and Therapy is an international, peer-reviewed, open access journal focusing on the pathological basis of all cancers, potential targets for therapy and treatment protocols employed to improve the management of cancer patients. The journal also focuses on the impact of management programs and new therapeutic agents and protocols on patient perspectives such as quality of life, adherence and satisfaction. The manuscript management system is completely online and includes a very quick and fair peer-review system, which is all easy to use. Visit http://www.dovepress.com/ testimonials.php to read real quotes from published authors. 